DOI: $10.3901 / J M E .2015 .17 .052$

\title{
不同重力环境下含间隙铰位置和碰撞力*
}

\author{
李仕华 ${ }^{1}$ 冯伟波 ${ }^{1}$ 秦 利 ${ }^{2}$ 刘福才 ${ }^{2}$ 徐继龙 ${ }^{1}$
}

(1. 燕山大学河北省并联机器人与机电系统实验室 秦皇岛 066004;

2. 燕山大学工业控制工程河北省重点实验室 秦皇岛 066004)

摘要: 分别以销轴和两维指向机构为研究对象, 结合 “接触-分离” 两状态模型, 采用多体系统动力学理论, 得到含间隙铰 的多体系统动力学方程。基于该方程, 采用动力学仿真软件 ADAMS 对含间隙铰的动力学特性进行分析, 得出了销轴在重力 场和微重力场中不同转速下销轴中心的运动轨迹及销轴与套筒碰撞力的变化规律。仿真结果表明: 重力释放对含间隙铰的运 动轨迹、振动、穿刺深度、碰撞力、未端精度等都具有较大的影响。研究成果对含间隙机构的设计具有重要价值和意义。 关键词: 间隙铰; 重力; 多体系统动力学; 动力学特性; 两维指向机构

中图分类号: V11

\section{Effect of Different Gravity Environment on the Position and Collision Force of Joint with Clearance}

\author{
LI Shihua $^{1}$ FENG Weibo $^{1}$ QIN Li $^{2}$ LIU Fucai $^{2} \quad$ XU Jilong ${ }^{1}$ \\ (1. Hebei Provincial Key Laboratory of Parallel Robot and Mechatronic System, Yanshan University, \\ Qinhuangdao 066004; \\ 2. Key Lab of Industrial Computer Control Engineering of Hebei Province, Yanshan University, \\ Qinhuangdao 066004)
}

\begin{abstract}
The multibody system dynamic equations of pin and two-dimensional pointing mechanism with clearance are obtained by using "contact-separation" two-state model and the multibody system dynamic theory. Based on these equations, the dynamics characteristics are analyzed by using dynamic simulation software ADAMS. The trajectories center pin and collision force change regularities are obtained in gravity field and micro gravity field at different speeds. The simulation results show that the release of gravity has great influence on the trajectories, vibration, penetration depth, collision force and end-effector precision of clearance joint. The results have important value and great significance for clearance mechanism design.
\end{abstract}

Key words: clearance joint; gravity; multibody system dynamic theory; dynamics characteristics; two-dimensional pointing mechanism

\section{0 前言}

世界各国越来越重视太空的利用和开发, 不断 地利用航天器和太空探测仪器开展近地空间和宇宙 的科学实践活动, 如发射了各种各样的太空望远镜、 星载天线和光学遥感器等, 开展对地球及外太空的 研究。其中美国 1990 年发射的著名的 Hubble 空间 望远镜, 是目前最先进的空间望远镜。太空具有失 重、高真空等特性, 航天器机械结构的运动副间隙 处的运动行为与重力下时不同, 若按重力下的运动 行为去设计航天器, 将会对航天器在太空中的有效

* 国家高技术研究发展计划(军口 863 计划)和河北省自然科学基金 (F2015203362)资助项目。20140927 收到初稿, 20150416 收到修改稿
使用带来影响 ${ }^{[1]}$ 。

航天器机械结构的运动副间陌 ${ }^{[2]}$ 主要包括动配 合设计需要而产生的间隙、运动副设计制造过程中 误差产生的间隙以及摩擦磨损形变导致的间隙。若 不能掌握运动副间隙和接触力变化规律, 将会对航 天器的设计带来不利影响。

自 20 世纪 70 年代以来, 国内外学者对间隙机 构开展了相关研究并取得了许多研究成果 ${ }^{[3-8]}$ 。何柏 岩等 ${ }^{[9]}$ 建立了含铰链间隙的刚柔机械臂动力学模 型，考虑了间隙和弹性变形及其约束方程的耦合问 题。FLORES 等 ${ }^{[10]}$ 提出了考虑间隙和润滑关节的机 械系统动力学分析, 论证了润滑油膜对接触冲击力 的影响。北京大学陈滨等 ${ }^{[11-12]}$ 研究了含铰接间隙与 杆件柔性的空间伸展机构单元的动力学建模与计算 
模拟, 得到了铰接间隙对伸展机构关节力、振动等 影响的一系列规律。

本文通过销轴和两维指向机构模型, 运用多刚 体系统动力学理论知识, 建立了多铰接机械系统的 间隙判别方式、动力学方程、接触力模型。通过 ProE、ADAMS 软件建立实际模型, 进行了不同转 速, 不同间隙大小, 不同重力环境下销轴中心轨迹 和碰撞力的仿真分析。

\section{1 多刚体系统动力学方程}

\section{1 销轴转动 “碰撞” 及其判别方式}

首先建立间隙铰销轴与套筒之间的接触碰撞 模型, 就是否发生碰撞、碰撞时的冲击力及碰撞方 程进行确定。图 1 建立了间隙铰销轴与套筒之间的 碰撞模型。
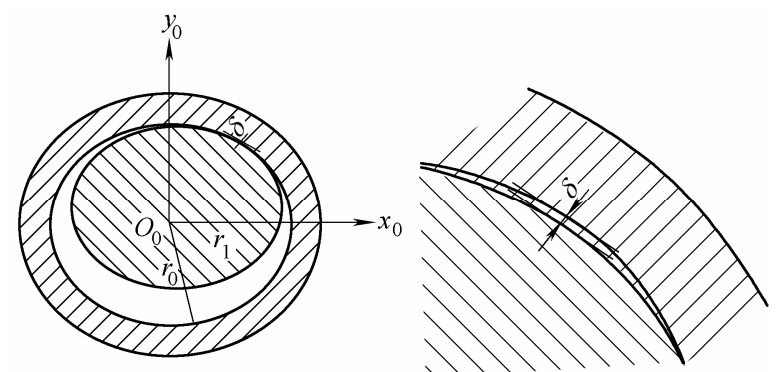

图 1 间隙铰碰撞模型及其局部放大图

图 1 中, $r_{0} 、 r_{1}$ 分别为套筒和销轴的半径, $O_{0}$ 、 $O_{1}$ 分别为位于套筒质心的固定坐标系原点和位于 销轴质心的相对坐标系原点。 $x_{1} 、 y_{1}$ 为销轴质心在 固定坐标系 $X$ 轴和 $Y$ 轴上的投影值, $\delta$ 为穿刺深 度。令 $\Delta r$ 为理想状态时的间隙值, 则 $\Delta r=r_{0}-r_{1}$, 那么穿刺深度 $\delta$ 可以表示为

$$
\delta=\sqrt{x_{1}^{2}+y_{1}^{2}}-\Delta r
$$

销轴和套筒处于何种状态可以通过 $\delta$ 大小进行 判别

$$
\begin{cases}\delta<0 & \text { 分离 } \\ \delta=0 & \text { 临界接触 } \\ \delta>0 & \text { 接触分离 }\end{cases}
$$

\section{2 含间隙铰的多体系统动力学方程}

含间隙运动副运动过程中会产生三维的接触 力, 接触力分解为法向碰撞力 $F_{n}$ 、切向摩擦力 $F_{f}$ 及 摩擦力引入的阻力矩 $M_{f}$ 。

此状态利用逻辑函数表示为

$$
F^{i}= \begin{cases}F_{n}+F_{f} & \delta \geqslant 0 \\ 0 & \delta<0\end{cases}
$$

在 $\delta<0$ 即运动副分离时, 多铰接机械系统动力 学方程为

$$
\left\{\begin{array}{l}
\boldsymbol{M} \ddot{\boldsymbol{q}}+\boldsymbol{\Phi}_{q}^{\mathrm{T}} \boldsymbol{\lambda}=\boldsymbol{Q} \\
\boldsymbol{\Phi}(\boldsymbol{q}, t)=0
\end{array}\right.
$$

式中 $\boldsymbol{q}$ 一广义坐标列阵;

$\boldsymbol{M}$ 一广义质量阵;

$\boldsymbol{\Phi}_{q}$ ——约束雅可比矩阵;

$\lambda$ - Lagrange 乘子列阵;

$\boldsymbol{Q}$ 一广义力阵;

$\boldsymbol{\Phi}(\boldsymbol{q}, t)$ ——约束矩阵。

在 $\delta \geqslant 0$ 即运动副接触穿刺时, 刚体之间发生了 碰撞, 产生了三维的碰撞力, 此时, 多铰接机械系 统动力学方程 ${ }^{[13]}$ 为

$$
\left\{\begin{array}{l}
\boldsymbol{M} \ddot{\boldsymbol{q}}+\boldsymbol{\Phi}_{q}^{\mathrm{T}} \lambda+\overline{\boldsymbol{\Phi}}_{q}^{\mathrm{T}} \bar{\lambda}=\boldsymbol{Q}+\boldsymbol{Q}_{A}+\boldsymbol{Q}_{B} \\
\boldsymbol{\Phi}(q, t)=0 \\
\overline{\boldsymbol{\Phi}}(q, t)=0
\end{array}\right.
$$

式中 $\boldsymbol{Q}_{A}+\boldsymbol{Q}_{B}$ 一间隙铰内力广义力阵。

2 销轴位置变化规律仿真研究

\section{1 间隙铰模型及参数}

间隙铰模型及参数见表 1 和图 2、图 3。销轴、 套筒材料均为 45 钢。

表 1 间隙铰模型的具体参数

\begin{tabular}{cc}
\hline 参数 & 数值 \\
\hline 销轴半径 $/ \mathrm{mm}$ & 50 \\
销轴质量 $/ \mathrm{kg}$ & 15 \\
套筒半径 $/ \mathrm{mm}$ & 55 \\
套筒质量 $/ \mathrm{kg}$ & 28 \\
\hline
\end{tabular}

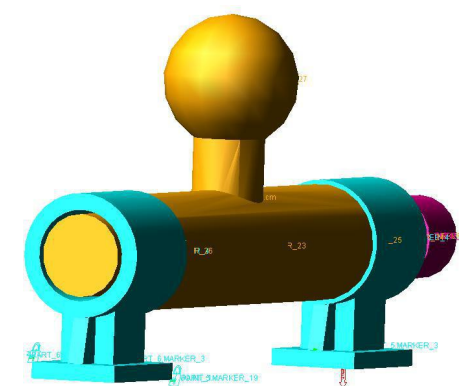

图 2 简化模型示意图

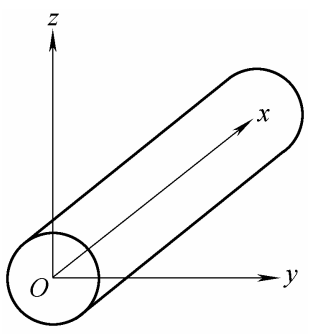

图 3 简化模型坐标系 
表 1 是轻载荷大间隙下间隙铰模型的具体参 数, 为使间隙更加直观对其进行了放大处理。

\section{2 重力释放前不同转速下销轴中心的变化规律}

图 4 为销轴中心在 “低速 $1000 \%$, 中速 $1500 \%$, 高速 $2500 \%$, 下的轨迹图。初始状态下 销轴中心和套筒中心重合, 坐标系如图 3 所示: 坚 直方向(即重力方向)为 $Z$ 轴方向, 水平方向为 $Y$ 轴 方向, 与轴线平行的方向为 $X$ 轴方向, 具体模型如 图 2 所示。三种转速默认为低速、中速、高速的状 态是相对具体结构、质量等参数而言的。

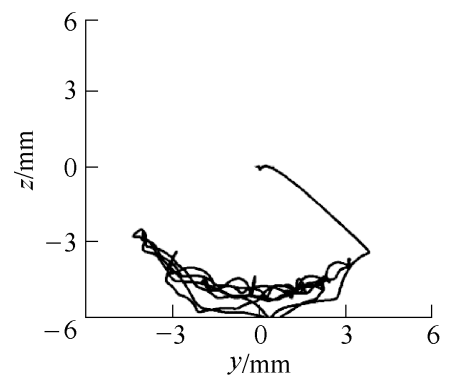

(a) 转速 $1000 \%$

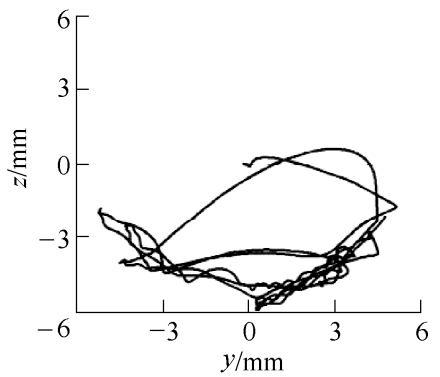

(b) 转速 $1500 \%$

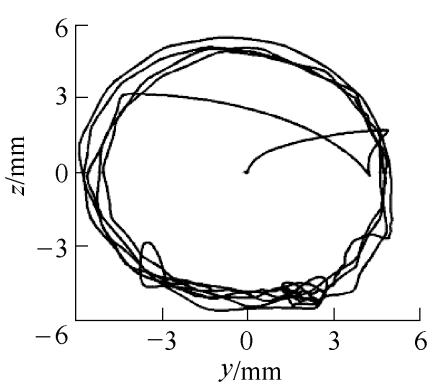

(c) 转速 $2500 \%$

图 4 销轴中心在重力下不同转速时运动轨迹

从图 4 可知, 销轴中心运动轨迹都在以套筒中 心处半径 $5 \mathrm{~mm}$ 的圆形区域内。在重力场中, 当销 轴以 “相对低速” 转动时, 由于向心力不足以克服 重力的影响, 销轴运动集中在圆形区域底端; 当销 轴以 “相对中速” 转动时, 其运动轨迹有沿圆形内 壁上升的趋势, 销轴转动到顶端后, 出现的碰撞现 象更为普遍, 穿刺范围、深度有所增加; 当销轴以 “相对高速” 转动时, 圆周运动的向心力克服重力的 影响, 其运动轨迹集中在圆周边界处, 运动基本稳定。
不同的转速, 销轴运动轨迹不同, 这是因为物 体所受到的加速度大小与合外力成正比，销轴绕套 筒内壁运动, 所受法向合力提供销轴圆周转动的向 心力, 切向力产生切向加速度, 能改变销轴的转速。 由于不同转速下, 向心力对重力的影响不同, 因此 形成销轴轨迹随销轴转速变化而变化。

\section{3 重力释放后四种转速下销轴中心的变化规律}

微重力环境中销轴中心在不同转速下的变化 规律如图 5 所示。从图 5 可以看出, 未运动时销轴 中心与套筒中心重合, 运动开始后, 销轴撞向套筒

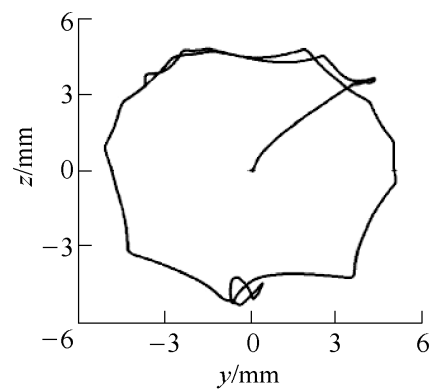

(a) 转速 $360 \%$

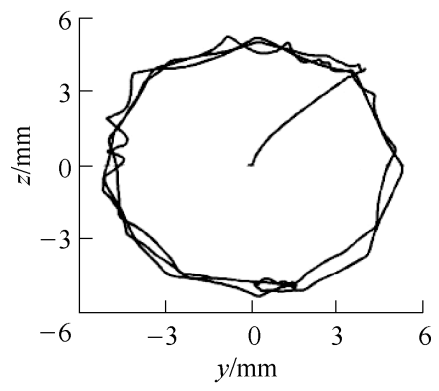

(b) 转速 $1000 \%$

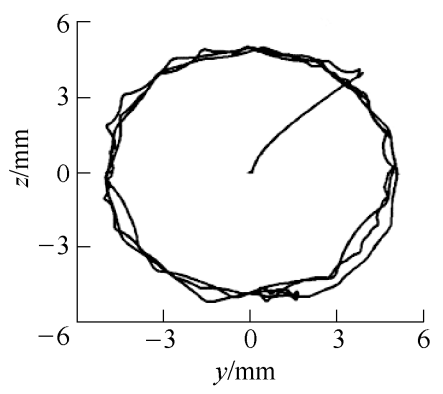

(c) 转速 $1500 \%$

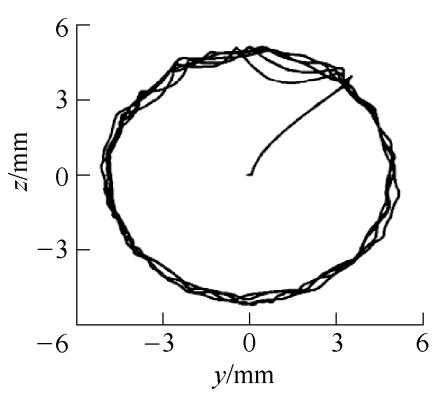

(d) 转速 $2500 \%$

图 5 销轴中心在失重下不同转速时运动轨迹 
内壁, 略微超出固定间隙值 $5 \mathrm{~mm}$, 出去部分为穿 刺深度, 然后再以 “边振动边转动” 的形式运动, 由于没有重力的影响, 运动轨迹趋向于圆周内壁。 在微重力场中当销轴以四种不同转速运动时, 运动 轨迹皆在圆周内壁上; 转速越快, 轨迹越密集于内 壁, 穿刺点越多; 高速转动时, 微重力下销轴的运 动轨迹与重力下的轨迹的基本相同, 但是有重力情 况下运动更为稳定，波动量略微收敛。

在微重力场中, 销轴转动受到套筒对销轴的作 用力, 产生切向加速度和法向加速度。销轴运动过 程中伴随有瞬时碰撞, 使其产生边振动边自转的耦 合情况。由于缺少掉落的过程, 所以碰撞力产生的 概率及大小会减弱。

3 高精度两维指向摆镜轴心的变化 规律仿真研究

\section{1 两维指向机构的具体参数及模型}

以上对含间隙销轴的中心轨迹规律进行了分 析, 下面参考文献[14]中高精度两维指向摆镜结构 及其参数, 按表 2 参数进行动力学仿真。U 型架材 料为铝合金, 转轴材料为 45 钢。

\section{表 2 两维指向机构部分参数}

\begin{tabular}{cc}
\hline 参数 & 数值 \\
\hline 俯仰角 $/\left(^{\circ}\right)$ & $-5 \sim 50$ \\
方位角 $\left(^{\circ}\right)$ & $-45 \sim 45$ \\
静摩擦因数 & 0.03 \\
动摩擦因数 & 0.01 \\
穿刺系数 & 0.1 \\
间隙值 $/ \mathrm{mm}$ & 0.5 \\
\hline
\end{tabular}

图 7 针对图 6 主机做了适量的简化处理。仿真 时, 两维指向机构的俯仰轴转速较低, 接近空间机 构实际转速, 轴承间隙值和轴颈较接近于实际大 小; 动静摩擦因数大小按在铰链中加入固体润滑剂 取值。

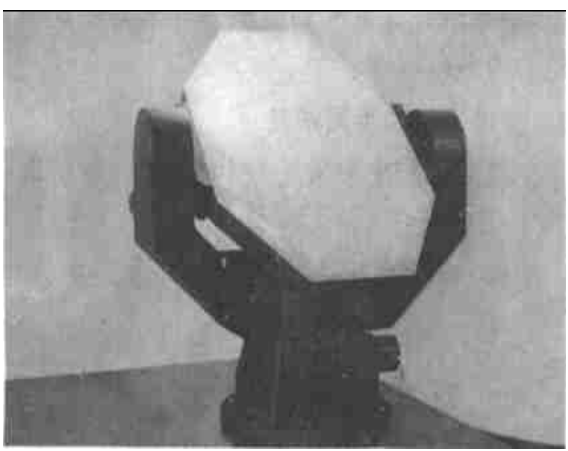

图 6 文献[14]中主机外形照片

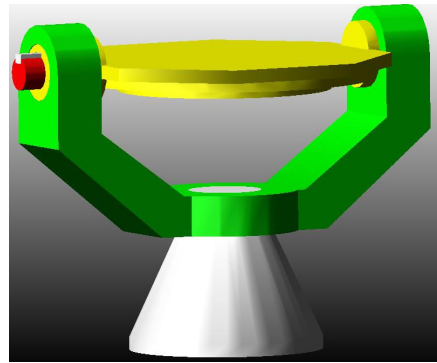

图 7 仿真分析模型

\section{2 重力释放前销轴中心沿 $Y 、 Z$ 轴及 $Y Z$ 平面的 运动轨迹}

从图 8 可知, 沿 $Y$ 轴方向销轴轴心的运动轨迹 的理论值在零线上下 $0.5 \mathrm{~mm}$ 的平行线内部, 但是 实际运动轨迹出现了总体下移，在零线上下 $0.5 \mathrm{~mm}$ 之外存在穿刺, 由于重力的作用使得下端比上端密。 由于水平方向不受重力作用销轴中心的运动轨迹基 本对称, 但在理论曲线之外也存在一定的穿刺深度。

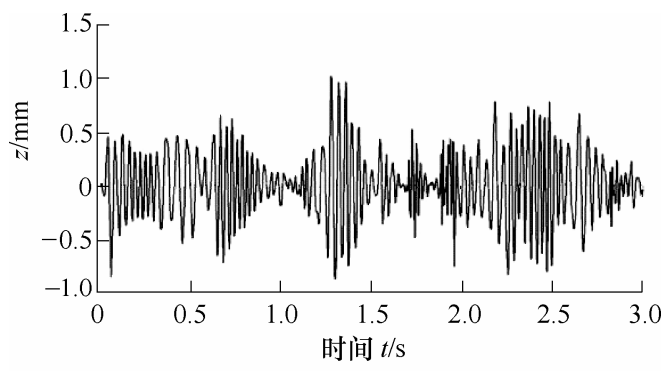

(a) $Y$ 轴方向的运动轨迹图

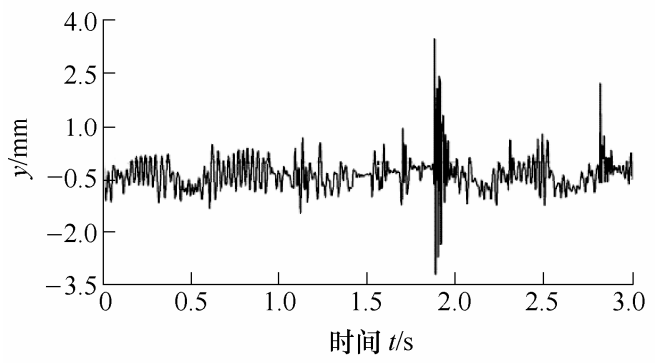

(b) $Z$ 轴方向的运动轨迹图

图 8 重力场中销轴中心的运动轨迹图

从图 9 可知, 销轴中心的理想运动轨迹为以半 径为 $0.5 \mathrm{~mm}$ 的圆, 但实际情况却是由杂乱无章的 线条组成的不规则区域。且该区域总体因为重力而 整体“下沉”，圆区域之外有穿刺现象。

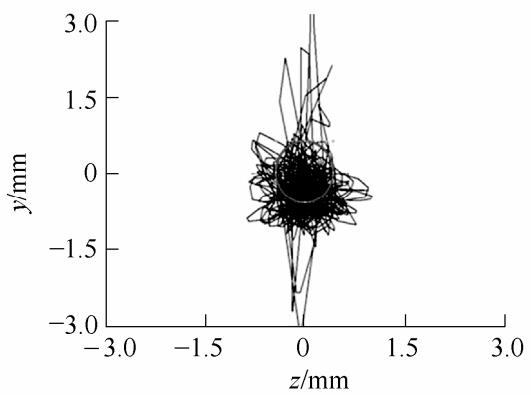

图 9 重力场中销轴中心在 $Y Z$ 平面的轨迹图 


\section{3 重力释放后销轴中心沿 $Y 、 Z$ 轴及 $Y Z$ 平面的 运动轨迹}

从图 10 可知, 在微重力场中, 由于没有重力, 销轴中心沿 $Y$ 轴方向和 $Z$ 轴方向的轨迹都关于零线 轴对称, 没有出现 “总体下沉”。相比重力场, 微重 力场中销轴中心的运动轨迹离套筒中心更近, 对应 的穿刺深度更小。

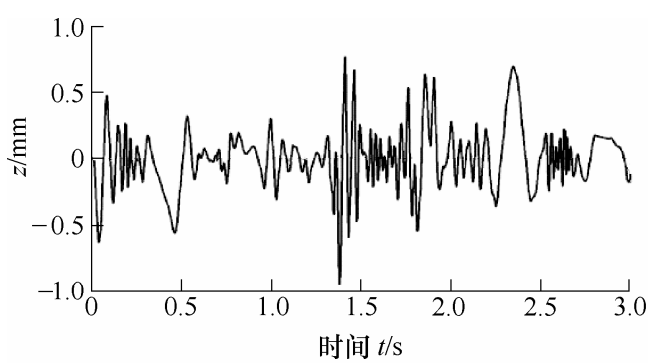

(a) $Y$ 轴方向的运动轨迹图

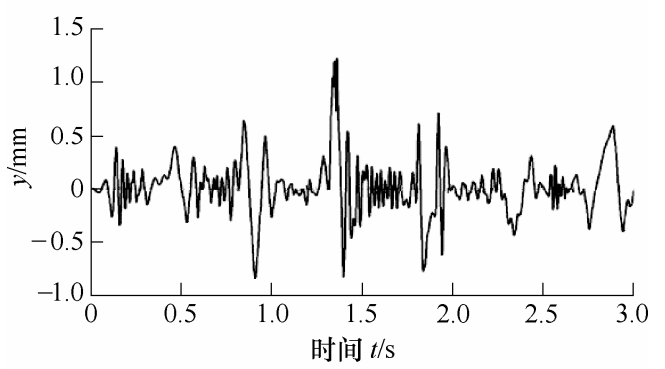

(b) Z 轴方向的运动轨迹图

图 10 微重力场中销轴中心的运动轨迹图

从图 11 可知, 里面圆圈所示区域为不考虑穿刺 时, 销轴中心的运动轨迹。微重力场中, 销轴中心 在 $Y Z$ 平面的轨迹没有 “下沉”, 但其运动轨迹仍然 是由杂乱无章的线条组成的区域，这个区域分布在 零线两侧, 基本对称。圆外出现穿刺现象, 相比重 力场, 微重力场中的穿刺范围小很多, 即微重力场 中的接触力比重力场小很多。

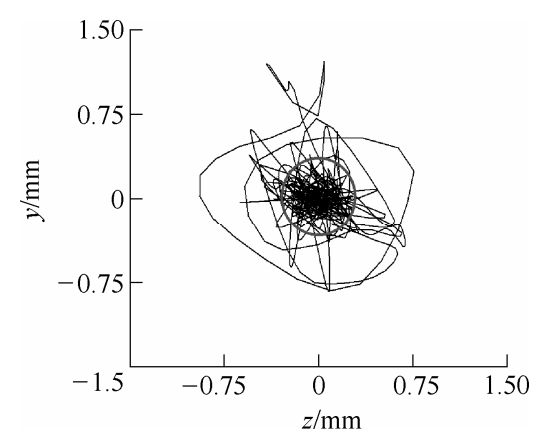

图 11 微重力场中销轴中心在 $Y Z$ 平面的轨迹图

4 接触力变化规律仿真研究

4.1 重力释放前两维指向机构销轴接触力变化规律 从图 12 可知, 由于初始给定销轴中心与套筒
中心重合, 在驱动力的作用下, 销轴做离心运动, 瞬间撞击套筒内壁，从而出现瞬时碰撞力。总体来 看销轴的接触力比较稳定, 只在 $1.25 \mathrm{~s}$ 和 $2.0 \mathrm{~s}$ 时刻 出现瞬时冲击力, 其他时间呈现阻尼振荡的现象, 逐渐达到一个稳定值。除此之外, 由于重力的效应 使得 $Z$ 轴方向上的接触力幅值明显要大于 $X$ 轴和 $Y$ 轴方向的接触力幅值。

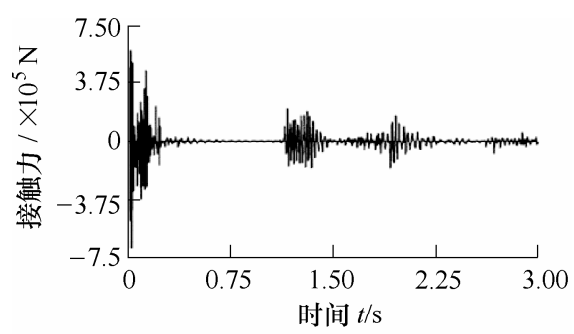

(a) $X$ 轴方向受力图

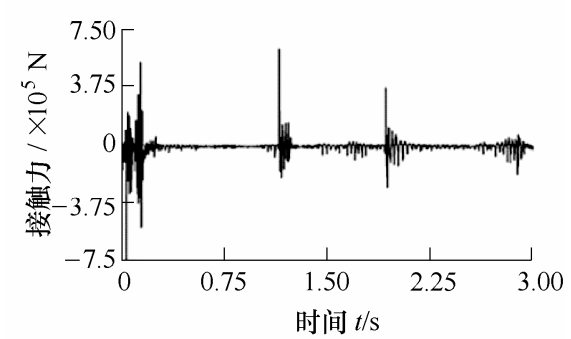

(b) $Y$ 轴方向受力图

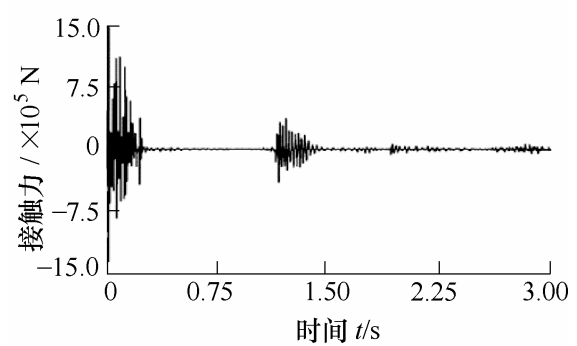

(c) $Z$ 轴方向受力图

图 12 重力场中销轴受力图

\section{2 重力释放后两维指向机构销轴接触力变化规律}

从图 13 可知, 微重力场中, 由于初始状态销 轴中心与套筒中心重合, 销轴运动开始阶段也出现 了瞬时碰撞。在 $1.15 \mathrm{~s}$ 时刻销轴的接触力出现很小 的突变, 其原因在于该时刻达到了系统的固有频率, 振动加强, 出现瞬时碰撞力。在经历短时间的振荡 后, 销轴的碰撞力逐渐趋于稳定。此外初始运转时 销轴产生很大的瞬时碰撞力, 这会对机构的稳定性 和可靠性带来不利影响。

\section{3 重力场中不同转速两维指向机构销轴接触力 变化规律}

从图 14 可知, 瞬时冲击力在有的转速下存在, 有的转速下不存在; 存在瞬时冲击力时接触力总体 比较稳定，局部有瞬时增大的力; 不存在瞬时冲击 


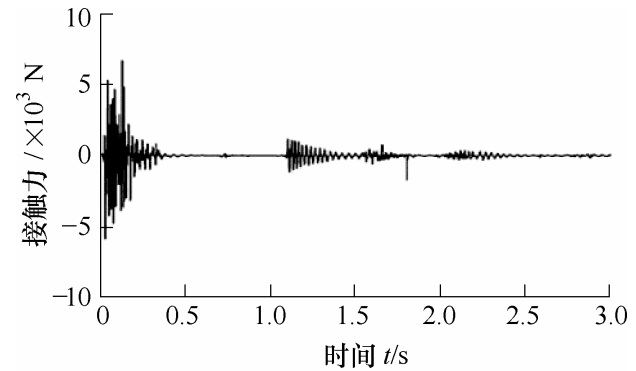

(a) $X$ 轴方向受力图

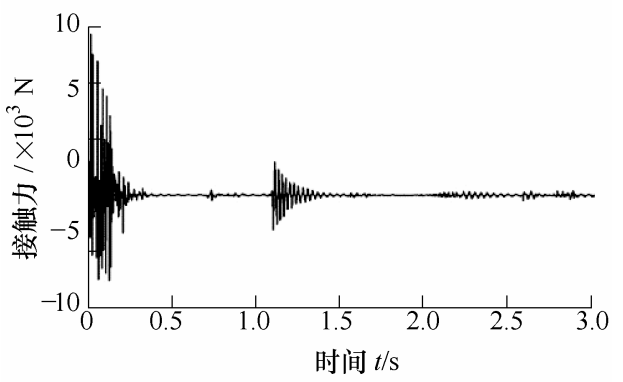

(b) $Y$ 轴方向受力图

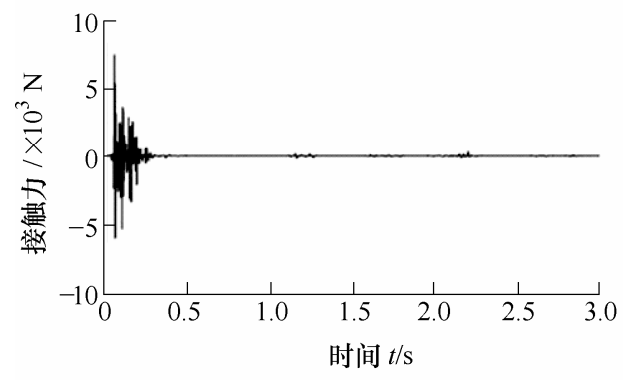

(c) $Z$ 轴方向受力图

图 13 微重力场中销轴受力图

力时接触力呈现阻尼振荡的现象, 逐渐达到一个稳 定值。之所以出现上述现象, 是因为间隙存在下销 轴是以自转、振动、爬升的形式运动, 当销轴从下 端运动到上面临界点后, 由于重力效应, 销轴跌落 并与套筒发生撞击, 从而产生瞬时碰撞力。此外, 销轴的最大接触力随转速的增加呈现先减小后增大 的趋势。在相同的转速周期, 出现瞬时碰撞力的时 间不同, 其原因在于驱动力、重力等力的耦合效应 使销轴达到合外力最大时对应的时间不一致。

\section{5 结论}

本文基于接触-分离模型, 分析了销轴间隙处的 状态及其变化规律, 并根据多体动力学理论, 建立 了含间隙机构的动力学方程。利用 ADAMS 进行了 仿真分析, 得出了不同重力环境下销轴中心的运动 轨迹以及销轴与套筒的接触力变化规律。

(1) 重力场中, 不同转速时销轴运动轨迹集中 在不同区域, 失重下不同转速时销轴运动区域几乎 相同。

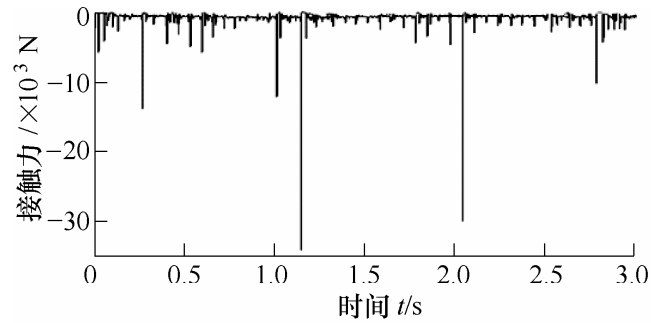

(a) 转速 $50 \sin 400 t$

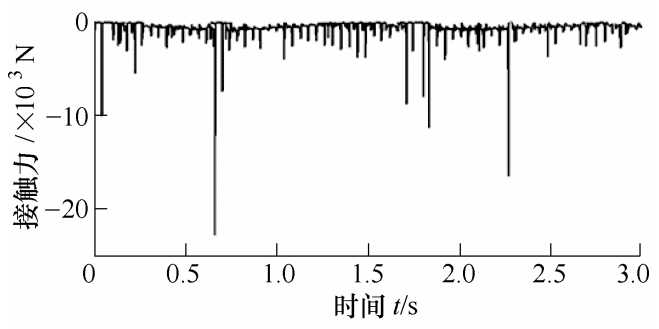

(b) 转速 $75 \sin 400 t$

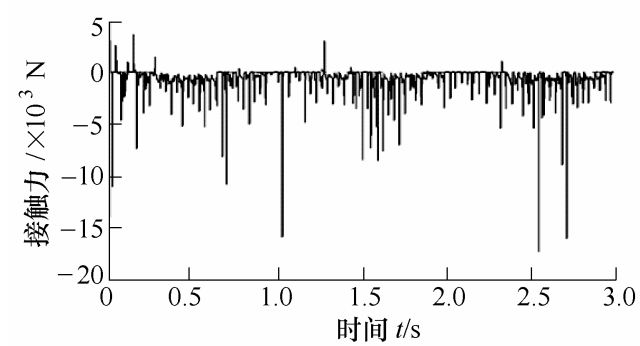

(c) 转速 $100 \sin 400 t$

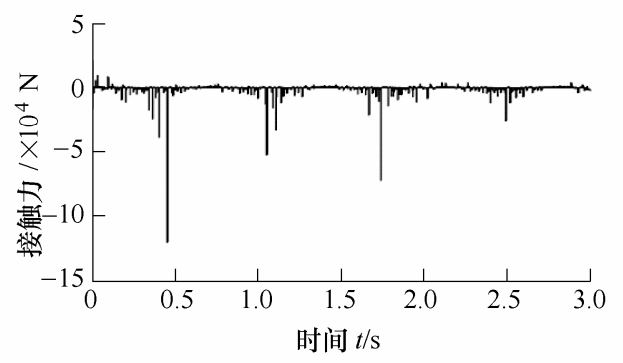

(d) 转速 $150 \sin 400 t$

图 14 重力场中不同转速时销轴受力图

（2）重力场中, 两维指向机构的运动轨迹在重 力方向上总体下移, 但在非重力方向上是关于中间 零线轴对称的。

(3) 重力场中，转速周期相同，不同幅值时, 销轴产生瞬时碰撞的时间不相同。

(4)重力场和微重力场中, 转速由低速变到高速 的过程中, 销轴中心的轨迹均出现了由稳定到不稳 定再到稳定变化情况。

(5) 相比重力场, 微重力场中销轴中心的运动 范围和受力更小。

\section{参 考 文 献}

[1] 阎绍泽，陈鹿民，季林红，等. 含间隙铰的机械多体系 统动力学模型 [J]. 振动工程学报, 2003, 16(3): 290-294. 
YAN Shaoze, CHEN Lumin, JI Linhong, et al. Dynamic modeling of multibody system with clearance joints[J]. Journal of Vibration Engineering, 2003, 16(3): 290-294.

[2] 白争锋. 考虑铰间间隙的机构动力学特性研究[D]. 哈 尔滨：哈尔滨工业大学，2011.

BAI Zhengfeng. Research on dynamic characteristics of mechanism with joint clearance[D]. Harbin : Harbin Institute of Technology, 2011.

[3] 靳春梅, 邱阳, 㚞灵, 等. 基于 FMD 理论的间隙机构 动力学研究 $[\mathrm{J}]$. 机械工程学报, 2001, 37(7): 18-22. JIN Chunmei, QIU Yang, FAN Ling, et al. Dynamics reserch of mechanism with clearences based on FMD theory[J]. Chinese Journal of Mechanical Engineering, 2001, 37(7): 18-22.

[4] LANKARANI H M, NIKRAVESH P E. A contact force model with hysteresis damping for impact analysis of multibody systems[J]. Journal of Mechanical Design, 1990, 112: 369-376.

[5] LIU Caishan, ZHANG Ke, YANG Rei. The FEM analysis and approximate model for cylindrical joints with clearances $[\mathrm{J}]$. Mechanism and Machine Theory, 2007, 42: 183-197.

[6] ZHU Jianmin, TING K. Uncertainty analysis of planar and spatial robots with joint clearances[J]. Mechanism and Machine Theory, 2000，35(9): 1239-1256.

[7] 许立新, 李永刚, 李充宁, 等. 轴承间隙及柔性特征对 机构动态误差的影响分析[J]. 机械工程学报, 2012, 48(7): $30-36$.

XU Lixin, LI Yonggang, LI Chongning, et al. Effects of bearing clearance and flexibility on the dynamic errors of mechanisms[J]. Journal of Mechanical Engineering, 2012, 48(7): 30-36.

[8] WITTENBURG J. Dynamics of systems of rigid bodies [M]. Stuttgart B G: Teubner, 2012.

[9] 何柏岩, 王树新, 张颖. 含铰链间隙的刚-柔机械臂动 力学模型 $[J]$. 动力学与控制学报, 2004, 2(2): 70-73.

HE Baiyan, WANG Shuxin, ZHANG Ying. A dynamic model of the mechanical arm with joint clearance[J]. Journal of Dynamics and Control, 2004, 2(2): 70-73.

[10] FLORES P, AMBROSIO J, CLARO J, et al. A study on dynamics of mechanical systems including joints with clearance and lubrication[J]. Mechanism and Machine Theory, 2006, 41: 247-261.
[11] 陈滨, 潘寒萌. 含铰接间隙与杆件柔性的空间伸展机构 单元的动力学建模与计算模拟, 第一部分: 动力学建 模[J]. 导弹与航天运载技术，1997(1): 27-37.

CHEN Bin, PAN Hanmeng. Dynamic modeling and simulation of unit of space deployable mechanism with joint clearance and link flexibility, Part I : Dynamic modeling of the system[J]. Missiles and Space Vehicles, 1997(1): 27-37.

[12] 陈滨, 潘寒萌. 含铰接间隙与杆件柔性的空间伸展机构 单元的动力学建模与计算模拟, 第二部分: 系统动态 特性的计算模拟结果 $[\mathrm{J}]$. 导弹与航天运载技术, 1997(3): 33-40.

CHEN Bin, PAN Hanmeng. Dynamic modeling and simulation of unit of space deployable mechanism with joint clearance and link flexibility, Part II : Computational simulation results on dynamic behavior of the system[J]. Missiles and Space Vehicles, 1997(3): 33-40.

[13] 李海阳, 吴德隆, 张永. 机动武器系统的含间隙动力学 研究, 下篇: 系统仿真 $[J]$. 兵工学报, 2002, 23(4): 433-437.

LI Haiyang, WU Delong, ZHANG Yong. Dynamic analysis of mobile weapon systems with clearances, Part III: System simulation[J]. Acta Armamentarii, 2002, 23(4): 433-437.

[14] 郝斌, 王胡顺. 高精度两维指向摆镜的研制 [J]. 光子学 报，2001，30(1): 117-119.

HAO Bin, WANG Hushun. Development of high precision two-dimensional sewing mirror[J]. Acta Photonica Sinica, 2001, 30(1): 117-119.

作者简介: 李仕华(通信作者), 男, 1966 年出生, 博士, 博士研究生导 师。主要研究方向为并联机器人技术及理论。

E-mail: shli@ysu.edu.cn

冯伟波, 男, 1987 年出生。主要研究方向为二维指向机构精度与应用。 E-mail: weibof@126.com

秦利, 女, 1984 年出生, 博士研究生。主要研究方向为空间机器人控制 技术。

E-mail: q1@ysu.edu.cn

刘福才, 男, 1966 年出生, 博士, 博士研究生导师。主要研究方向为非 线性系统模糊辨识与预测控制, 空间机器人控制技术, 新能源系统计算 机控制。

E-mail:1fc@ysu.edu.cn

徐继龙, 男, 1989 年出生。主要研究方向为并联机器人技术及理论。 E-mail: xjlong_good@163.com 